\title{
FP-LMTO investigation of the structural, electronic and magnetic properties of Heusler compounds $\mathrm{Ru}_{2} \mathrm{CrZ}(\mathrm{Ge}, \mathrm{Sn}, \mathrm{Si})$
}

\author{
S. Bahlouli ${ }^{1}$, Z. Aarizou ${ }^{1}$, M. Elchikh ${ }^{1, a}$, and G. Vergoten ${ }^{2}$ \\ 1 Laboratoire de Physique des Matériaux et des Fluides, Département de Physique, Université des Sciences et \\ de la Technologie d'Oran-MB, BP 1505 Oran El Mnaouer, Algeria \\ 2 Unité de Glycobiologie Structurelle et Fonctionnelle, UMR CNRS/USTL n 8576-IFR 118, Université des Sci- \\ ences et Technologies de Lille, Batiment C9, 59655 Villeneuve d'Ascq cedex, France
}

\begin{abstract}
We report structural and magnetic properties as well as band structures and density of states (DOS) of full Heusler $\mathrm{Ru}_{2} \mathrm{CrSi}, \mathrm{Ru}_{2} \mathrm{CrGe}$ and $\mathrm{Ru}_{2} \mathrm{CrSn}$. This was performed in the frame work of self-consistent first-principle calculations, using the Full-Potential Linearized Muffin Tin Orbital (FP-LMTO) method based on the Generalized Gradient Approximation (GGA), to investigate the structure and magnetic properties through the calculation of the electronic structure, equilibrium lattice constant and magnetic properties. Our results will show that our three Full-Heusler compounds are antiferromagnets.
\end{abstract}

\section{Introduction}

Heusler compounds have attracted renewed interest because they have been expected to be new candidates for future applications. Full Heusler compounds are ternary intermetallics with general formula $\mathrm{X}_{2} \mathrm{YZ}$ first discovered a century ago by Heusler [1]. These materials crystallize in L2 1 structure with space group FmN3m. This structure consists of four interpenetrating FCC (Face Centered Cubic) sublattices with the following Wyckoff coordinates: X(1/4, 1/4, 1/4), (3/4, $3 / 4,3 / 4), \mathrm{Y}(0,0,0), \mathrm{Z}(1 / 2,1 / 2,1 / 2)$, along with the primitive translation vectors $\mathbf{A}, \mathbf{B}$ and $\mathbf{C}$ :

$\mathbf{A}(0,1 / 2,1 / 2), \mathbf{B}(1 / 2,0,1 / 2), \mathbf{C}(1 / 2,1 / 2,0)$. Typical $\mathrm{X}$ and $\mathrm{Y}$ site elements are transition metal elements and $\mathrm{Z}$ may be any one of the large number of s-p elements belonging to the IIIB-VB group. Most of these alloys are ferromagnetic at room temperature, though a large number of Heusler alloys are available as ferromagnetic systems, many alloys show other interesting properties like semiconducting and antiferromagnetic behaviors, for a recent review see for instance the paper by C. Felser et al. [2]. Kanomata et al. [3] have grown crystals of the type $\mathrm{Ru}_{2} \mathrm{MnZ}$, where $\mathrm{Z}$ stands for Si, Ge and Sn. Gotoh et al. [4] have shown that these alloys are antiferromagnets with Neel temperatures near room temperature, and Ishida et al. [5] using first-principles calculations demonstrated that the ground state is antiferromagnetic (AFM). Okada et al. succeeded in synthesizing $\mathrm{Ru}_{2} \mathrm{CrGe}$ and $\mathrm{Ru}_{2} \mathrm{CrSn}$, and found that $\mathrm{Ru}_{2} \mathrm{CrGe}$ is an antiferromagnet with Néel temperature $T_{N}=13 \mathrm{~K}$ and $\mathrm{Ru}_{2} \mathrm{CrSn}$ shows a spin-glass-like behavior below $T_{g}=7 \mathrm{~K}[6]$. Mizutani et al. [7,8] and Brown et al. [9] have studied the fullHeusler alloys $\mathrm{Ru}_{2-x} \mathrm{Fe}_{x} \mathrm{Si}$ and $\mathrm{Ru}_{2-x} \mathrm{Fe}_{x}$ Ge respectively and investigated the phase transition to ferro-

\footnotetext{
${ }^{a}$ e-mail: elchikh65@gmail.com
}

magnetic state where Fe are partially or totally substituted for Ru. In the present contribution we review our most recent results on the magnetic behavior as well as the electronic properties of $\mathrm{Ru}_{2} \mathrm{CrZ}(\mathrm{Z}=\mathrm{Si}, \mathrm{Ge}$ and $\mathrm{Sn}$ ) obtained from first-principles electronic structure calculations. In section 2 , we briefly describe the calculational model and method. The results and discussions are presented in Section 3, and a brief summary is given in Section 4.

\section{Method of Calculations}

To fulfill the present calculation, we have applied the FP-LMTO method $[10,11]$ as embedded in LmtART MStudio MindLab 7 code developed by Savrasov [11, 12]. The exchange and correlation potential was calculated using GGA Perdew Wang (PW) scheme [13]. In the present study, the different muffin tin radius $R_{\mathrm{MT}}$ used are given as follows (in a.u.):

$\mathrm{Ru}_{2} \mathrm{CrSi}: \mathrm{Ru}(2.477), \mathrm{Cr}(2.38), \mathrm{Si}(2.38)$.

$\mathrm{Ru}_{2}$ CrGe: $\mathrm{Ru}(2.509), \mathrm{Cr}(2.413), \mathrm{Si}(2.413)$.

$\mathrm{Ru}_{2} \mathrm{CrGe:} \mathrm{Ru}(2.535), \mathrm{Cr}(2.535), \mathrm{Si}(2.535)$. In these calculations, the FP-LMTO basis set consists of the $4 d^{7} 5 s^{1}$ and $3 d^{5} 4 s^{1}$ states for the transition elements $\mathrm{Ru}$ and $\mathrm{Cr}$ respectively. For the $\mathrm{Z}$ atoms, the valence states are $3 \mathrm{~s}^{2} 3 \mathrm{p}^{2}$ of $\mathrm{Si}, 4 \mathrm{~s}^{2} 4 \mathrm{p}^{2}$ of Ge or $5 \mathrm{~s}^{2} 5 \mathrm{p}^{2}$ of $\mathrm{Sn}$. But the filled states $4 \mathrm{~d}^{10}$ of $\mathrm{Sn}$ and $3 \mathrm{~d}^{10}$ of Ge may also be considered as valence states, that are the default input in the code since the energy needed to separate semi-core and valence electrons was set to above $-2 \mathrm{Ry}$ from the vacuum zero, which results in treating of Si, Ge and $\mathrm{Sn}$ semi-core electrons as valence electrons. The charge density and potential are expanded in spherical harmonics inside the spheres up to $\operatorname{lmax}=6$ and Fourier transformed in the interstitial region. The maximum value of angular momentum 
$l \max =6$ is also taken for wave function expansion inside the atomic spheres. The lattice constants used in the present study, were obtained by minimizing the total energy $E_{t o t}$ with respect to the experimental lattice parameters. The energy convergence criterion was set to $10^{-5}$ Ry. For $k$-space integration over the Brillouin zone, we found that our calculations converge for a $10 \times 10 \times 10$ tetrahedron mesh [14]. The energy cut-off and the number of plane waves used in our calculations are, respectively: (80.70 Ry; 9204) for $\mathrm{Ru}_{2} \mathrm{CrGe}$, (75.40Ry; 9204) for $\mathrm{Ru}_{2} \mathrm{CrSn}$ and (84.09Ry; 9204) for $\mathrm{Ru}_{2} \mathrm{CrSi}$. In our study the magnetic unit cell being twice that of the unit cell used in crystallography (defined above) if the AFM phase is considered. For this AFM phase, we adopt the so called FCC-AFM type II order. More precisely, we consider the new unit structure defined by: $\mathrm{X}(1 / 4,1 / 4,1 / 4, \uparrow),(3 / 4,3 / 4,3 / 4, \uparrow)$, $\mathrm{Y}(0,0,0, \uparrow), \mathrm{Z}(1 / 2,1 / 2,1 / 2, \uparrow), \mathrm{X}(5 / 4,5 / 4,5 / 4, \downarrow)$, $(7 / 4,7 / 4,7 / 4, \downarrow), \mathrm{Y}(1,1,1, \downarrow), \mathrm{Z}(3 / 2,3 / 2,3 / 2, \downarrow)$, $\mathbf{A}^{\prime}(1 / 2,1 / 2,1), \mathbf{B}^{\prime}(1 / 2,1,1 / 2), \mathbf{C}^{\prime}(1,1 / 2,1 / 2)$.

The $\uparrow$ and $\downarrow$ are set for the magnetic moment orientation along the positive and negative [001] direction respectively (positive and negative $z$ direction). Hence, the magnetic moments on the atoms are ferromagnetically coupled on the (111) planes and antiferromagnetically coupled in adjacent planes. In Fig. 1, we show this magnetic arrangement only for the case of $\mathrm{Cr}$ atoms with similar magnetic arrangement on $\mathrm{Ru}$ and Ge atoms. In fact, this arrangement (AFM type II) has been revealed experimentally for $\mathrm{Ru}_{2} \mathrm{CrGe}[9]$.

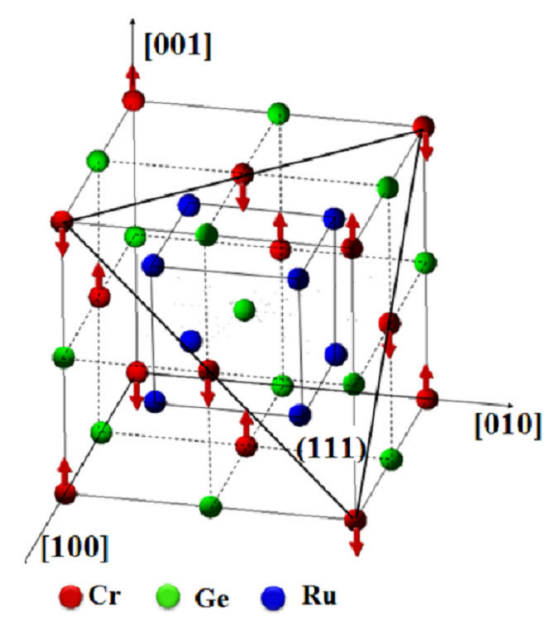

Fig. 1. Magnetic moment arrangement on $\mathrm{Cr}$ atoms.

\section{Results and discussion}

\subsection{Structural optimization}

First, to carry out the lattice constants which give the lowest total energy, we performed structural optimizations on our three Heusler compounds of interest, $\mathrm{Ru}_{2} \mathrm{CrSi}, \mathrm{Ru}_{2} \mathrm{CrGe}$ and $\mathrm{Ru}_{2} \mathrm{CrSn}$, for the Nonmagnetic (NM), ferromagnetic (FM) and AFM phases. For the three Heusler compounds, the results of structural optimization are shown in Fig. 2. One can clearly notice that the total energy differences $\Delta E=E_{A F M}-$ $E_{F M}$ are negative, thus all our compounds $\mathrm{Ru}_{2} \mathrm{CrZ}$ are stable in the AFM phase which confirm the experimental results. In order to calculate the ground states properties, we computed the total energies for several lattice constants and fitted them with the empirical Murnaghan equation of state [15] the values of the optimized lattice constants are given in Table 1 . We note that our calculated lattice constants for $\mathrm{Ru}_{2} \mathrm{CrGe}$ and $\mathrm{Ru}_{2} \mathrm{CrSn}$ are in agreement with the experimental values. To the best of our knowledge, no experimental data have been reported for $\mathrm{Ru}_{2} \mathrm{CrSi}$.

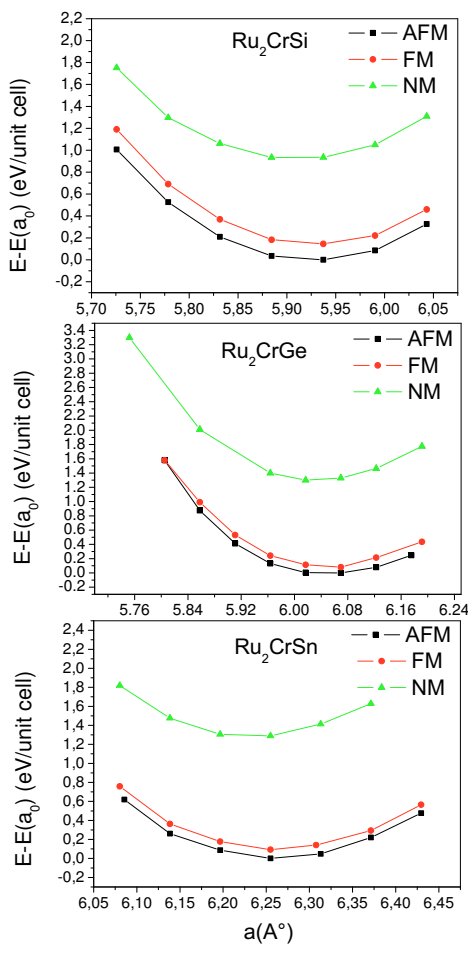

Fig. 2. Structural optimization: $E\left(a_{0}\right)$ being the ground state energy.

It is clear that the theoretical lattice constant and the energy differences $\Delta E$ in $\mathrm{Ru}_{2} \mathrm{CrZ}$ ( $\mathrm{Z}=\mathrm{Si}$, Ge and $\mathrm{Sn})$ increases with the increasing atomic number of $\mathrm{Si} \rightarrow \mathrm{Ge} \rightarrow \mathrm{Sn}$ due to the increasing size of atomic radius.

\subsection{Electronic and magnetic properties}

The calculated band structures of the AFM state of $\mathrm{Ru}_{2} \mathrm{CrZ}$, being quite similar, so only the $\mathrm{Ru}_{2} \mathrm{CrGe}$ electronic structure is displayed in Fig. 3. Furthermore, our three compounds being antiferromagnets, then the electronic properties of the two spin-channels are similar consequently one spin-channel has been reported in Fig. 3. Our three compounds present a gap along $\Gamma-X$, i.e. in the $(\Delta)$ direction and the Fermi 
Table 1. Equilibrium lattice constants $a_{0}(\AA)$ and total energy differences $\Delta E\left(\mathrm{eV} /\right.$ unit cell) for $\mathrm{Ru}_{2} \mathrm{CrZ}$ compounds.

\begin{tabular}{llll}
\hline Compound & Calculation & $a_{0}$ & $\Delta E$ \\
\hline $\mathrm{Ru}_{2} \mathrm{CrSi}$ & Our work & 5.93 & -0.1414 \\
& Experimental & & \\
& Other [7] & 5.94 & \\
$\mathrm{Ru}_{2} \mathrm{CrGe}$ & Our work & 6.05 & -0.110 \\
& Experimental [6,9] & 5.97 & \\
& Other & 6.02 & \\
$\mathrm{Ru}_{2} \mathrm{CrSn}$ & Our work & 6.26 & -0.0984 \\
& Experimental [6] & 6.19 & \\
& Other [16] & 6.233 & \\
\hline
\end{tabular}

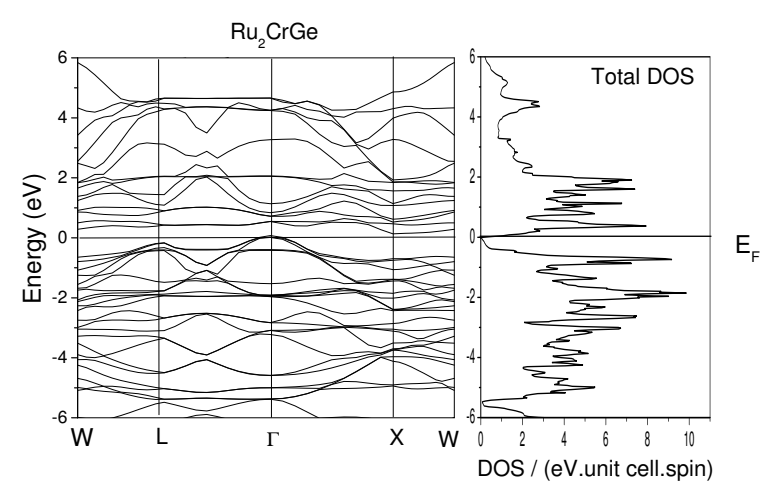

Fig. 3. Electronic structure of $\mathrm{Ru}_{2} \mathrm{CrGe}$

energy of these materials is just at the top of the valence band. Calculated energy gap (in eV) are 0.35 for $\mathrm{Ru}_{2} \mathrm{CrSi}, 0.14$ for $\mathrm{Ru}_{2} \mathrm{CrGe}$ and 0.02 for $\mathrm{Ru}_{2} \mathrm{CrSn}$. We note that our Heusler Z-atoms have an influence on the energy gap, therefore, the gap decreases as the number of valence $\mathrm{Z}$ increases. The partial density of states (PDOS) of $\mathrm{Ru}_{2} \mathrm{CrGe}$ as a function of energy is shown in Fig. 4, the PDOS of $\mathrm{Ru}_{2} \mathrm{CrSi}$ and $\mathrm{Ru}_{2} \mathrm{CrSn}$ being almost the same. The high DOS around $\mathrm{E}_{F}$ is associated with hybridization of $\mathrm{Ru}(4 \mathrm{~d})$ and $\mathrm{Cr}(3 \mathrm{~d})$ electrons. We notice that the $\mathrm{Cr}$ compound exhibits a considerably larger gap compared with the $\mathrm{Ru}$ compound. In order to understand the influence of $\mathrm{Z}$ element on the gap width we plotted the PDOS of Z elements in Fig 5. The energy of (p) electrons is strongly dependent on the $\mathrm{Z}$ atom in $\mathrm{Ru}_{2} \mathrm{CrZ}$, so the hybridization between $(p)$ electrons with different energy and the (d) electrons affects the formation of the energy gap. The calculated values of the $\mathrm{Ru}$ and $\mathrm{Cr}$ local moments are given as follows $\left(\mu_{\mathrm{B}}\right)$ :

$\mathrm{Ru}_{2} \mathrm{CrSi}: \mathrm{Ru}(0.045), \mathrm{Cr}(2.173)$,

$\mathrm{Ru}_{2} \mathrm{CrGe:} \mathrm{Ru}(0.094), \mathrm{Cr}(2.291)$,

$\mathrm{Ru}_{2}$ CrGe: $\mathrm{Ru}(0.078), \operatorname{Cr}(2.483)$. We can see that the local magnetic moments of $\mathrm{Cr}$ become large with increasing the atomic number of the $\mathrm{Z}$ atoms but also the lattice constant has an influence on the magnetic properties, on the other hand the $\mathrm{Ru}$ atom carries negligible local moment as it was deduced from experiment [9]. In Fig 6, we plotted the PDOS of $\mathrm{Ru}(4 \mathrm{~d})$ atom and $\operatorname{Cr}(3 \mathrm{~d})$ atom for both spin states (with pos- itive magnetic moment), we can see the large splitting between spin-up and spin-down PDOS at Fermi energy of Cr compared to the low equivalent splitting PDOS of Ru. However, the individual magnetic moment of $\mathrm{Cr}$ atoms found in our study, is about fifty percent greater than the value of $1.45 \mu_{\mathrm{B}}$, estimated experimentally in the case of $\mathrm{Ru} 2 \mathrm{CrGe}$ at $5 \mathrm{~K}$ [9], which indicates that itinerant exchange mechanism alone can not explain this difference. It is interesting to note that when $\mathrm{Ru}$ is replaced by other elements like Co [17, 18] or Mn [19], Heusler alloys exhibit half metallic ferromagnetic behavior. The conduction band minimum consists mostly of $\mathrm{Ru}(4 \mathrm{~d}), \mathrm{Cr}(3 \mathrm{~d})$ and smaller contribution of $\mathrm{Z}(\mathrm{p})$ orbitals; the valence band maximum is composed of $\mathrm{Cr}(3 \mathrm{~d}), \mathrm{Ru}(4 \mathrm{~d})$ and $\mathrm{Z}(\mathrm{p})$ electrons. Moreover, $\mathrm{Z}$ atoms create four fully occupied bands, comprising of one $\mathrm{Z}(\mathrm{s})$ and three $\mathrm{Z}(\mathrm{p})$ bands. This $\mathrm{Z}(\mathrm{s})$ is very low in energy and also well separated from other bands, on the other hand, Z(p) bands hybridize with $\mathrm{p}$ electron $\mathrm{Ru}$ and $\mathrm{Cr}$, but they $(\mathrm{Z}(\mathrm{p})$ bands) are also low energy bands. The d- states of the transition metal atoms ( $\mathrm{Ru}$ and $\mathrm{Cr}$ ) extend from occupied and unoccupied states and hybridize with each other. The widely spread d states are mainly due to the strong hybridization of the $(3 \mathrm{~d})$ metals. In ad-

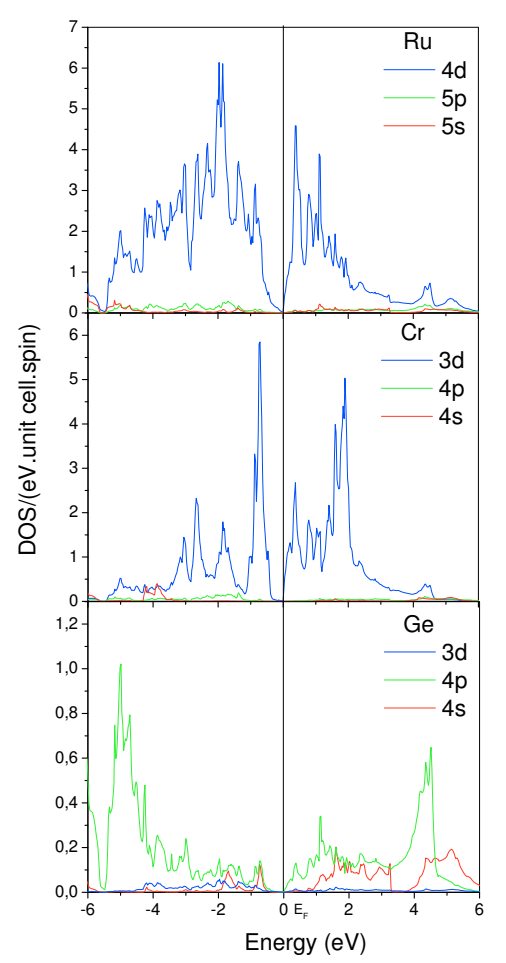

Fig. 4. $\mathrm{PDOS}$ of $\mathrm{Ru}_{2} \mathrm{CrGe}$.

dition, if In $\left(5 \mathrm{~s}^{2} 5 \mathrm{p}^{1}\right)$ is chosen to be the $\mathrm{Z}$ element instead of $\mathrm{Si}$ or Ge or Sn, the situation changes drastically. In fact, we found that $\mathrm{Ru}_{2} \mathrm{CrIn}$ is ferrimagnetic Heusler compound with semi-metallic behavior. Furthermore, when we take into account of the correlation effect by using GGA $+U$ method with appropriate values for $U$ and $J$ parameters, we found this has no 


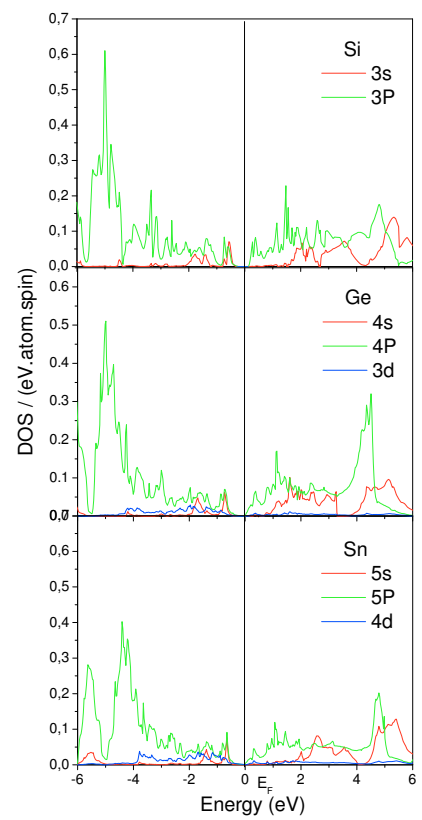

Fig. 5. Compared PDOS of Z elements.

significant effect on the semi-conducting behavior in the case of $\mathrm{Ru}_{2} \mathrm{CrZ}(\mathrm{Z}=\mathrm{Si}, \mathrm{Ge})$ while it seems that the $\mathrm{Ru}_{2} \mathrm{CrSn}$ compound is more sensitive since its energy gap is somewhat very small $(0.02 \mathrm{eV})$ as our first calculations indicate but no suitable values $(U, J)$ have been obtained yet. It also is interesting to mention that the half metallic behavior is recovered within the $\mathrm{GGA}+U$ method in the case of $\mathrm{Ru}_{2} \mathrm{CrIn}$, more details can be found in our forthcoming paper. Let us note

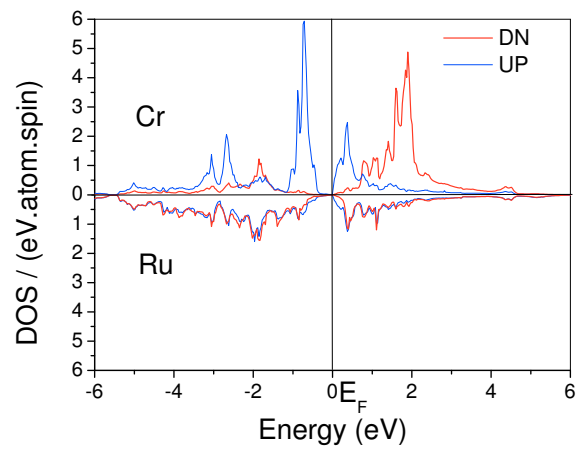

Fig. 6. Compared PDOS of $\mathrm{Ru}(4 \mathrm{~d})$ and $\mathrm{Cr}(3 \mathrm{~d})$.

that the half metallic ferromagnetic behavior can be present in $\mathrm{Ru}_{2-x} \mathrm{Fe} x \mathrm{CrSi}$ for a particular range of $\mathrm{Fe}$ concentration $x$ starting at $x=0.5[7,8]$. The antiferromagnetic, along with a spin-glass behavior of these Heusler compounds cannot be simply explained by itinerant exchange mechanism and need more theoretical effort.

\section{Summary}

In the present work, we have performed first principles calculations of structural, electronic and magnetic properties of $\mathrm{Ru}_{2} \mathrm{CrZ}(\mathrm{Z}=\mathrm{Si}, \mathrm{Ge}$ and $\mathrm{Sn}$ ) full Heusler alloys within the GGA scheme. We find that our compounds have AFM ground states which are in good agreement with the experimental results. The electronic properties show that these compounds have a semiconductor behavior with indirect gap along $\Gamma-X$. We found that the $\mathrm{Z}$ elements have an influence on the electronic properties as well as on the magnetic properties. Therefore the energy gap decreases as the number of valence $\mathrm{Z}$ increases also the theoretical values of the $\mathrm{Cr}$ local moments become larger with increasing of the $\mathrm{Z}$ atomic number. The high DOS around $\mathrm{E}_{F}$ is associated with hybridization of $\mathrm{Ru}(4 \mathrm{~d})$ and $\mathrm{Cr}(3 \mathrm{~d})$ electrons.

\section{References}

1. F. Heusler, Verh. Dtsch. Phys. Ges. 5, (1903) 219.

2. T. Graf, C. Felser and S. S.P. Parkin, Progress in Solid State Chemistry 39, (2011) 1.

3. T. Kanomata, M. Kikuchi, H. Yamauchi, and T. Kaneko, Jpn. J. Appl. Phys. 32 Suppl 32-33, (1993) 292.

4. M. Gotoh, M. Phashi, T. Kanomata, and Y. Yamaguchi, Physica B 306, (1995) 213.

5. S. Ishida, S. Kashiwagi, S. Fujii, and S. Asano, Physica B 140, (1995) 210.

6. H.Okada et al., App. Phys.Lett. 92, (2008) 062502. H.Okada et al., Journal of Physics: Conference Series 150, (2009) 042153.

7. S. Mizutani, S. Ishida, S. Fujii, S. Asano, Mater. Trans. 47, (2006) 25.

S. Ishida, S. Mizutani, S. Fujii, S. Asano, Mater. Trans. 47, (2006) 31.

8. M. Hiroi et al., Phys. Rev. B 79, (2009) 224423.

M. Hiroi et al., Journal of Physics: Conference Series 150, (2009) 042058.

9. P.J Brown et al., J. Phys.: Condens.Matter 20, (2008) 455201.

10. S.Y. Savrasov and D. Savrasov, Phys.Rev. B 46 , (1992) 12181.

11. S.Y. Savrasov, Phys. Rev. B 54, (1996) 16470.

12. http://physics.ucdavis.edu/ mindlab

13. J. P. Perdew, S. Burke and M. Ernzerhof, Phys. Rev. Lett. 77, (1996) 3865.

14. P.E. Blchl, O. Jepsen, O.K. Andersen, Phys. Rev. B 49, (1994) 16223.

15. F.D Murnaghan, Proc. Nat. Acad. Sci. of U.S.A 30 (1944) 244.

F. Birch, Phys. Rev. 71 (1947) 809.

16. M. Gilleen, R. Dronskowski, Journal Of Computational Chemistry, 30, (2009) 1290.

17. H.C Kandpal, G. H Fecher and C. Felser; J. Phys. D: Appl. Phys 40 (2007) 1507.

18. Z. Aarizou, A. Akriche, S. Bahlouli and M. Elchikh, (Unplished work).

19. H. Luo et al., J. Magn. Magn. Mater. 320 (2008) 421. 